\title{
Experimental verification of horizontal two-dimensional modified mild-slope equation model
}

\author{
Kyung Doug Suh ${ }^{\mathrm{a},{ }^{*}}$, Changhoon Lee ${ }^{\mathrm{b}}$, Young-Hyun Park ${ }^{\mathrm{c}, * *}$, Tae Hwan Lee ${ }^{\mathrm{d}}$ \\ ${ }^{a}$ School of Civil, Urban and Geosystem Engineering \& Research Institute of Marine Systems Engineering, \\ Seoul National University, Seoul 151-742, South Korea \\ ${ }^{\mathrm{b}}$ Department of Civil and Environmental Engineering, Sejong University, Seoul 143-747, South Korea \\ ${ }^{\mathrm{c} S}$ School of Civil, Urban and Geosystem Engineering, Seoul National University, Seoul 151-742, South \\ Korea \\ ${ }^{\mathrm{d}}$ Daewoo Corporation Engineering \& Construction, C.P.O. Box 8269, Seoul 100-095, South Korea
}

\begin{abstract}
In order to verify modified mild-slope equation models in a horizontal twodimensional space, a hydraulic experiment is made for surface wave propagation over a circular shoal on which water depth varies substantially. A horizontal two-dimensional numerical model is also constructed based on the hyperbolic equations that have been developed from the modified mild-slope equation to account for the substantial depth variation. Comparison between experimental measurements and numerical results shows that the modified mild-slope equation model is capable of producing accurate results for wave propagation in a region where water depth varies substantially, while the conventional mild-slope equation model gives large errors as the mild-slope assumption is violated.
\end{abstract}

Keywords: Hydraulic experiment; Mild-slope equation; Numerical model; Water waves; Wave propagation

\footnotetext{
*Corresponding author. Fax: +82-2-887-0349; E-mail: kdsuh@ snu.ac.kr

${ }^{* * *}$ Present address: Dept. of Civil Eng., Texas A\&M Univ., College Station, TX 77844, USA.
} 


\section{Introduction}

The mild-slope equation (abbreviated as MSE hereinafter) developed by Berkhoff (1972) has been widely used to compute the transformation of surface gravity waves in coastal regions. It has not only been used in its original form of elliptic equation but also provided the basic governing equation for the development of other wave propagation models such as parabolic model (Radder, 1979), hyperbolic model (Copeland, 1985) and angular spectrum model (Dalrymple et al., 1989).

The MSE assumes that the water depth varies slowly over a wavelength, that is, $|\nabla h| / k h<<1$, where $\nabla=$ horizontal gradient operator, $h=$ water depth, and $k=$ wave number. A few studies have attempted to extend the MSE to account for substantial depth variation. Kirby (1986) extended the mild-slope approximation to include rapidly varying, small amplitude deviations from a slowly varying topography and applied the resulting equation to a two-dimensional problem involving wave reflection from sinusoidal beds. Tsay et al. (1989) subsequently applied Kirby's extended equation to three-dimensional bedforms. O'Hare and Davies (1992) developed a new model to simulate wave transformation in rapid undulations by approximating the bed as a series of horizontal shelves. A similar technique was also used by Guazzelli et al. (1992) and Cho and Lee (2000).

Recently efforts have been made to improve the MSE by including the higher-order bottom effect terms proportional to the square of bottom slope and to the bottom curvature, which were neglected in the derivation of the MSE (Massel, 1993; Chamberlain and Porter, 1995; Suh et al., 1997; Chandrasekera and Cheung, 1997). Neglecting the evanescent wave modes, for a monochromatic wave, the equations of all these authors, in spite of different approaches of derivation, reduce to a same equation, which will be referred to as the modified mild-slope equation (abbreviated as MMSE hereinafter) in this paper as named by Chamberlain and Porter (1995). More recently, Lee et al. (1998) recasted the MMSE into the form of a pair of first-order equations, which constitute a hyperbolic system. All of these authors applied the MMSE to twodimensional problems involving wave reflection from sinusoidal beds or a submerged bar. On the other hand, Chandrasekera and Cheung (1997) applied the MMSE to three- 
dimensional bedforms. First they computed the wave transformation over a circular shoal for which the experimental data of Sharp (1968) are available. They compared the solutions of the MSE and the MMSE with the experimental data, but the difference between the MSE and the MMSE was not significant. Both solutions showed reasonable agreement with the experimental data, because the effects of the higher-order bottom effect terms are minor. To evaluate the significance of these terms, they performed a numerical experiment using circular shoals of different configuration, for which they found that the two solutions showed significant difference.

Chadrasekera and Cheung (1997) performed only a numerical experiment for the circular shoals for which the MSE and the MMSE showed significant difference. In the present study, we carry out a hydraulic experiment for wave transformation over one of the shoals used in the numerical experiment of Chadrasekera and Cheung. We also construct a horizontal two-dimensional numerical model based on Lee et al.'s (1998) hyperbolic equations that were developed from the MMSE. The experimental measurements are then compared with the numerical model results. In the following section, the hydraulic experiment for wave transformation over a circular shoal is described. In section 3, Lee et al.'s (1998) equations are briefly summarized and the finite difference formulation of the equations is made in a horizontal two-dimensional space. In section 4, the hydraulic experimental data are compared with the numerical model results. Comparison with the MSE model is also made to elucidate its limitation in the situations of substantial depth variation.

\section{Hydraulic experiment}

The experiment was conducted in the wave tank at the Coastal Engineering Laboratory of Seoul National University. The wave tank is $11 \mathrm{~m}$ wide, $23 \mathrm{~m}$ long, and 1 $\mathrm{m}$ high. The wave paddle is only $6 \mathrm{~m}$ wide, so guide walls were installed along the tank and wave absorbers at both ends of the tank as shown in Fig. 1. Waves are generated with a piston-type wave maker. The wave generation and data acquisition are controlled by a personal computer. Water surface displacement was measured with parallel-wire resistance-type wave gauges.

The circular shoal was patterned after that used in the numerical experiment of 
Chandrasekera and Cheung (1997). It was made by milling acrylic resin and was pasted on the bottom of the tank. The water depth on the shoal at a distance $r$ from the center is given by

$$
h=h_{0}-b\left[1-\left(\frac{r}{R}\right)^{2}\right]
$$

where $h_{0}=0.3 \mathrm{~m}$ is the water depth on flat bottom, $b=0.18 \mathrm{~m}$ is the height of the shoal at its center, and $R=0.45 \mathrm{~m}$ is the radius of the shoal. Since the water depth on the shoal is defined by a quadratic equation, the curvature $\nabla^{2} h$ over the shoal is constant as $4 b / R^{2}$, while the square of the slope $(\nabla h)^{2}$ ranges from zero at $r=0$ to $4 b^{2} / R^{2}$ at $r=R$.

The center of the shoal was located at $6 \mathrm{~m}$ apart from the wave maker. Wave measurements were made along the five lateral transects as well as the centerline as shown in Fig. 2. Surface elevation time histories were measured using an array of five wave gauges. They were spaced $5 \mathrm{~cm}$ apart in a steel frame that minimized the amount of interference from support legs. For the same wave condition, therefore, wave measurements were made three times along each lateral transect and nine times along the centerline at different locations. For the purpose of normalizing, the incident wave was measured at a point in front of the shoal where the effect of the shoal is minimal. The incident wave height was $3 \mathrm{~cm}$, and three different wave periods, 1.259, 0.791, and $0.636 \mathrm{~s}$, were used, which correspond to $k_{0} h_{0}=1.0,2.0$, and 3.0, respectively, where $k_{0}$ is the wave number on flat bottom. Even though the tests were made for regular waves, the wave measurements were made for $51.2 \mathrm{~s}$ at the sampling rate of $40 \mathrm{~Hz}$ to obtain 2048 data for each of the wave gauges. The wave height averaged over the sampling duration excluding the leading waves was then used for later analyses. Wave reflection from the shoal or the downwave absorber was negligible.

\section{Numerical model}




\subsection{Governing equations}

Following the procedure outlined in Copeland (1985), Lee et al. (1998) recasted the MMSE into the form of a pair of first-order equations, which constitute a hyperbolic system. The resulting equations are given as follows:

$$
\begin{aligned}
& \frac{\partial \eta}{\partial t}+\frac{1}{\frac{C_{g}}{C}-R_{1}(\nabla h)^{2}-R_{2} \nabla^{2} h} \nabla \cdot \mathbf{Q}=0 \\
& \frac{\partial \mathbf{Q}}{\partial t}+C C_{g} \nabla \eta=0
\end{aligned}
$$

where $\eta$ is the water surface elevation, $C$ and $C_{g}$ are the phase speed and group velocity, respectively, and $\mathbf{Q}$ is the volume flux defined as

$$
\mathbf{Q}=\frac{C C_{g}}{g} \nabla \tilde{\phi}
$$

Here $g$ is the gravitational acceleration, and $\tilde{\phi}$ is the velocity potential at mean water level, which is related to $\eta$ by

$$
\tilde{\phi}=-\frac{i g}{\omega} \eta
$$

where $i=\sqrt{-1}$ and $\omega$ is the wave angular frequency. In Eq. (2), $R_{1}$ and $R_{2}$ are the parameters determining the higher-order bottom effects, which are given by

$$
\begin{aligned}
& R_{1}=\frac{1}{\cosh k h}\left(W_{1} I_{1}+W_{2} I_{2}+W_{3} I_{3}+W_{4} I_{4}+W_{5} I_{5}+W_{6}\right) \\
& R_{2}=\frac{1}{\cos h k h}\left(U_{1} I_{1}+U_{2} I_{2}+U_{3} I_{3}\right)
\end{aligned}
$$


The expressions of $W_{i}, U_{i}$, and $I_{i}$ are given in the appendix. The wave number, $k$, is determined from the dispersion relationship given by

$$
\omega^{2}=g k \tanh k h
$$

Without the $(\nabla h)^{2}$ and $\nabla^{2} h$ terms, Eqs. (2) and (3) reduce to the Copeland's (1985) mild-slope hyperbolic equations.

\subsection{Finite difference method}

In order to numerically generate the waves at the open boundary, the internal wave generation technique (Larsen and Dancy, 1983; Madsen and Larsen, 1987) was adopted, which generates the waves along a line inside the model domain while permitting the waves propagating toward the wave generation line to freely pass across the line so that unwanted addition of wave energy in the model domain can be avoided. For the waves propagating in the positive $x$-direction, the value of $\eta^{*}$ as given below is added to the surface elevation at the wave generation line:

$$
\eta^{*}=2 \eta_{I} \frac{C \Delta t}{\Delta x}
$$

where $\eta_{I}$ is the water surface elevation of the incident wave, and $\Delta x$ and $\Delta t$ are the grid size in $x$-direction and time step, respectively.

Sponge layers are placed at both upwave and downwave boundaries to minimize wave reflection from the boundaries by dissipating wave energy inside the sponge layers. The thickness of the sponge layer, $S$, was taken as 2.5 times the local wavelength, which was found to reduce the amplitude of the incident wave to almost zero at the boundaries. In order to model the waves inside and outside the sponge layer continuously, Eq. (3) is modified as 


$$
\frac{\partial \mathbf{Q}}{\partial t}+C C_{g} \nabla \eta+\omega D_{s} \mathbf{Q}=0
$$

The damping coefficient, $D_{s}$, is given by

$$
D_{s}= \begin{cases}0, & \text { o u t s id p o n g a y e } \\ \frac{e^{d / s}-1}{e-1}, & \text { in s idsep o n g a y e }\end{cases}
$$

where $d$ is the distance from the starting point of the sponge layer.

Eqs. (2) and (10) are discretized by a leap-frog method in a staggered grid in both time and space, which yields

$$
\begin{aligned}
& \frac{\eta_{i, j}^{n+1 / 2}-\eta_{i, j}^{n-1 / 2}}{\Delta t}+\frac{\frac{P_{i+1 / 2, j}^{n}-P_{i-1 / 2, j}^{n}}{\Delta x}+\frac{Q_{i, j+1 / 2}^{n}-Q_{i, j-1 / 2}^{n}}{\Delta y}}{\left(\frac{C_{g}}{C}-R_{1}(\nabla h)^{2}-R_{2} \nabla^{2} h\right)_{i, j}}=0 \\
& \frac{P_{i-1 / 2, j}^{n+1}-P_{i-1 / 2, j}^{n}}{\Delta t}+\left(C C_{g}\right)_{i-1 / 2, j} \frac{\eta_{i, j}^{n+1 / 2}-\eta_{i-1, j}^{n+1 / 2}}{\Delta x}+\omega\left(D_{s}\right)_{i-1 / 2, j} P_{i-1 / 2, j}^{n}=0 \\
& \frac{Q_{i, j-1 / 2}^{n+1}-Q_{i, j-1 / 2}^{n}}{\Delta t}+\left(C C_{g}\right)_{i, j-1 / 2} \frac{\eta_{i, j}^{n+1 / 2}-\eta_{i, j-1}^{n+1 / 2}}{\Delta y}+\omega\left(D_{s}\right)_{i, j-1 / 2} Q_{i, j-1 / 2}^{n}=0
\end{aligned}
$$

where $P$ and $Q$ are the volume flux in the $x$-and $y$-direction, respectively [i.e., $\mathbf{Q}=(P, Q)]$. The variables are located in the rectangular grid shown in Fig. 3. In the preceding finite difference equations, the subscripts $i$ and $j$ increase in the $x$ - and $y$-direction, respectively, with their integral values at the center of each grid where the surface elevation $\eta$ is located. Superscript $n$ indicates elevation at time $n \Delta t$. It is apparent from the finite difference equations that the values of $P$ and $Q$ are calculated at a time $\Delta t / 2$ ahead of the corresponding values of $\eta$. All the values of $\eta, P$, and $Q$ at the initial time step are set to be zero. For the slow start of wave generation, Eq. (10) is multiplied by $\tanh (0.5 t / T)$ where $T$ is the wave period. In 
this study, the same grid spacing was used in $x$-and $y$-directions (i.e., $\Delta x=\Delta y$ ). The grid spacing $\Delta x$ was chosen to be $1 / 30$ of the wavelength on flat bottom. The time step was chosen for the Courant number $C_{r}=C \Delta t / \Delta x$ to be 0.2 so that a stable solution is guaranteed. In order to achieve a steady state of wave field, the waves were generated for $20 T$ or $30 T$ depending on the wave period. The numerical test conditions used are summarized in Table 1, in which $M$ and $N$ are the number of grid in the $x$-and $y$-direction, respectively.

The computational domain for the numerical model is shown in Fig. 4. The pure computing domain (from the wave generation line to the starting line of the downwave sponge layer) was taken to be the same as the domain in the hydraulic experiment $(16.75 \times 6.0 \mathrm{~m}$ as shown in Fig. 1). The sponge layers of thickness of 2.5 times the local wavelength were placed in front of both upwave and downwave model boundaries. The upwave sponge layer was separated by $\Delta x$ from the wave generation line. Along all the boundaries, perfect reflection is assumed, but at both upwave and downwave boundaries the reflected wave becomes negligible because the sponge layer significantly reduces the incoming wave energy.

\section{Comparison of experimental measurements and computational results}

Contour lines of the wave amplitude computed by the MMSE model relative to the incident amplitude are shown in Fig. 5 for different wave periods. The shoal boundary is indicated as a dashed circle. The crest of the shoal is located at $(6.0,3.0) \mathrm{m}$ with its radius of $0.45 \mathrm{~m}$. Wave focusing on or behind the shoal, generation of side depressions, and wave reflection by the shoal are clearly observed. It is also seen that the focal point moves downwave with decreasing wave period, probably because the wave refraction over the shoal becomes insignificant as the wave period decreases.

Results of the MMSE and MSE models along the centerline are shown in comparison with the experimental data in Fig. 6 for different wave periods. In each figure, the MMSE and MSE model results are indicated by solid and dashed lines, respectively, while bullets indicate experimental data. While fairly good agreement is observed between the MMSE model results and experimental data, the MSE model 
results deviate largely from the experimental data near and behind the shoal crest especially for the long-period wave $(T=1.259 \mathrm{~s}$ ) which feels the bottom more strongly. As the wave period becomes short, the focal point moves downwave and the maximum amplitude decreases, again because the shorter wave feels less the bottom so that the wave focusing becomes weak.

Results of the MMSE and MSE models along the lateral transects are shown in comparison with the experimental data in Figs. 7 to 9 for different wave periods. Acceptable agreement is observed between the MMSE model results and experimental data. The MSE model results, however, deviate largely from the experimental data as moving downwave from the shoal especially for the long-period wave for which the bottom effect is more significant. The MSE model over-predicts the wave amplitude in the focusing area, while under-predicting it at the side depressions. At the beginning of the shoal (i.e., at $x=-R$ ), there is almost no lateral variation of wave amplitude except for the longest wave ( $T=1.259 \mathrm{~s}$ ) for which the wave amplitude in front of the shoal slightly increases due to the wave reflection from the shoal (see Fig. 7a). As the wave passes over the shoal, the lateral variation of wave amplitude becomes large, and then it reduces as the wave propagates far downwave from the shoal. The location of the side depression also moves away from the centerline as the wave propagates downwave from the shoal.

Finally, in order to examine the relative importance of the bottom slope squared term and the bottom curvature term, we have tested the MSE model by including only either the bottom slope squared term or the bottom curvature term. A similar numerical test has been made by Chandrasekera and Cheung (1997) for the same circular shoal as that used in the present study. Our results are very similar to those of them. The results of the MSE model plus the bottom curvature term closely follow those of the MMSE model, while the inclusion of the bottom slope squared term does not significantly modify the results obtained by the MSE model. Without showing the results, we conclude that the bottom curvature term plays much more important role than the bottom slope squared term within the experimental conditions we used. The relative importance of these terms depends on not only bathymetry (i.e., bottom slope and curvature) but also relative water depth, having been described in other papers (e.g., Chandrasekera and Cheung, 1997; Lee et al., 1998). 


\section{Conclusions}

In order to verify the MMSE model in a horizontal two-dimensional space, we have made a hydraulic experiment for surface wave propagation over a circular shoal for which the effects of bottom slope squared term and bottom curvature term are not insignificant. We have also constructed a horizontal two-dimensional numerical model based on Lee et al.'s (1998) hyperbolic equations that were developed from the MMSE and thus include the higher-order bottom effect terms. By comparing the experimental measurements with the numerical results, we have shown that the MMSE model is capable of producing accurate results for wave propagation in a region where the depth varies substantially, while the MSE model gives large errors as the mild-slope assumption is violated.

\section{Acknowledgement}

This work was supported by the Brain Korea 21 Project.

\section{Appendix. Components of terms of $R_{1}$ and $R_{2}$}

$$
\begin{aligned}
& W_{1}=-2 \lambda+2 \frac{k_{h}}{k}+2 \frac{k h k_{h}}{\lambda}-4 \frac{\lambda h k_{h}}{k}+\frac{h k_{h h}}{k}+\frac{h^{2} k_{h}^{2}}{\lambda}-2 \frac{\lambda h^{2} k_{h}^{2}}{k^{2}} \\
& W_{2}=2 k-2 \frac{k_{h}}{\lambda}+2 h k_{h} \\
& W_{3}=2 k_{h}-\frac{k_{h h}}{\lambda}+2 \frac{h k_{h}^{2}}{k} \\
& W_{4}=-2 \frac{k k_{h}}{\lambda} \\
& W_{5}=-\frac{k_{h}^{2}}{\lambda} \\
& W_{6}=1+\frac{h k_{h}}{k}
\end{aligned}
$$




$$
\begin{aligned}
& U_{1}=1+\frac{h k_{h}}{k} \\
& U_{2}=-\frac{k}{\lambda} \\
& U_{3}=-\frac{k_{h}}{\lambda} \\
& I_{1}=\frac{1}{4 k}(2 k h+\mathrm{s} \text { i n } \mathrm{B} k h) \\
& I_{2}=\frac{1}{4 k}(\cos 2 k h-1) \\
& I_{3}=\frac{1}{4 k^{2}}\left(k h \cosh 2 k h-\frac{1}{2} \sinh 2 k h\right) \\
& I_{4}=\frac{1}{4 k^{2}}\left(k h \text { s in B } k h-\frac{1}{2} \cos 3 k h+k^{2} h^{2}+\frac{1}{2}\right) \\
& I_{5}=\frac{1}{4 k^{3}}\left[\left(k^{2} h^{2}+\frac{1}{2}\right) \sin \text { B } k h-k h \cos \mathbb{B} k h+\frac{2}{3} k^{3} h^{3}\right] \\
& k_{h}=\frac{\partial k}{\partial h}=-\frac{\lambda k}{\lambda h+\sinh ^{2} k h} \\
& k_{h h}=\frac{\partial^{2} k}{\partial h^{2}}=-\frac{\lambda k_{h}}{\lambda h+\sinh ^{2} k h}\left(2+\frac{k+h k_{h}}{\lambda} \sinh 2 k h\right) \\
& \lambda=\frac{\omega^{2}}{g}=k \tanh k h
\end{aligned}
$$

\section{References}

Berkhoff, J.C.W., 1972. Computation of combined refraction-diffraction. In: Proc. 13th Coastal Eng. Conf., pp. 471-490.

Chamberlain, P.G., Porter, D., 1995. The modified mild-slope equation. J. Fluid Mech. 291, 393-407.

Chandrasekera, C.N., Cheung, K.F., 1997. Extended linear refraction-diffraction model. J. Waterw. Port Coastal Ocean Eng. 123, 280-286.

Cho, Y.-S., Lee, C., 2000. Resonant reflection of waves over sinusoidally varying topographies. J. Coastal Res. 16, 870-876.

Copeland, G.J.M., 1985. A practical alternative to the mild-slope wave equation. Coastal 
Eng. 9, 125-149.

Dalrymple, R.A., Suh, K.D., Kirby, J.T., Chae, J.W., 1989. Models for very wide-angle water waves and wave diffraction. Part 2. Irregular bathymetry. J. Fluid Mech. 201, 299-322.

Guazzelli, E., Rey, V., Belzons, M., 1992. Higher-order Bragg reflection of gravity surface waves by periodic beds. J. Fluid Mech. 245, 301-317.

Kirby, J.T., 1986. A general wave equation for waves over rippled beds. J. Fluid Mech. $162,171-186$.

Larsen, J., Dancy, H., 1983. Open boundaries in short wave simulations - A new approach, Coastal Eng. 7, 285-297.

Lee, C., Park, W.S., Cho, Y.-S., Suh, K.D., 1998. Hyperbolic mild-slope equations extended to account for rapidly varying topography. Coastal Eng. 34, 243-257.

Madsen, P.A., Larsen, J., 1987. An efficient finite-difference approach to the mild-slope equation. Coastal Eng. 11, 329-351.

Massel, S.R., 1993. Extended refraction-diffraction equation for surface waves. Coastal Eng. 19, 97-126.

O'Hare, T.J., Davies, A.G., 1992. A new model for surface-wave propagation over undulating topography. Coastal Eng. 18, 251-266.

Radder, A.C., 1979. On the parabolic equation method for water-wave propagation. J. Fluid Mech. 95, 159-176.

Sharp, W.A., 1968. Water wave refraction over a submerged shoal. MEng. thesis, Univ. of Liverpool.

Suh, K.D., Lee, C., Park, W.S., 1997. Time-dependent equations for wave propagation on rapidly varying topography. Coastal Eng. 32, 91-117.

Tsay, T.K., Zhu, W., Liu, P. L.-F., 1989. A finite element model for wave refraction, diffraction, reflection, and dissipation. Applied Ocean Res. 11, 8-33. 
Table 1

Numerical test conditions

\begin{tabular}{cccccc}
\hline$k_{0} h_{0}$ & $\Delta x(\mathrm{~m})$ & $\Delta t(\mathrm{~s})$ & $C_{r}$ & $\begin{array}{c}\text { Duration of } \\
\text { wave generation }\end{array}$ & $(M, N)$ \\
\hline 1.0 & 0.062818 & 0.00834 & 0.2 & $20 T$ & $(388,139)$ \\
2.0 & 0.031406 & 0.00524 & 0.2 & $30 T$ & $(671,241)$ \\
3.0 & 0.020937 & 0.00420 & 0.2 & $30 T$ & $(908,325)$ \\
\hline
\end{tabular}




\section{Captions of figures}

1. Illustration of experimental setup.

2. Wave measurement transects.

3. Finite-difference grid.

4. Computational domain.

5. Amplitude contours relative to incident wave amplitude. (a) $T=1.259 \mathrm{~s}$, (b) $T=$ $0.791 \mathrm{~s},(\mathrm{c}) \mathrm{T}=0.636 \mathrm{~s}$.

6. Comparison of numerical model results with experimental data along centerline: solid line $=$ MMSE model; dashed line $=$ MSE model; $\bullet=$ experimental data. (a) $T=$ $1.259 \mathrm{~s}$, (b) $T=0.791 \mathrm{~s}$, (c) $T=0.636 \mathrm{~s}$.

7. Comparison of numerical model results with experimental data along lateral transects for the case of $T=1.259 \mathrm{~s}$ : solid line = MMSE model; dashed line = MSE model; $\bullet$ = experimental data. (a) $x=-R$, (b) $x=0$, (c) $x=R$, (d) $x=2 R$, (e) $x=3 R$.

8. Same as Fig. 7, but for $T=0.791 \mathrm{~s}$.

9. Same as Fig. 7, but for $T=0.636 \mathrm{~s}$. 